\title{
TÉCNICAS QUALITATIVAS EM ANÁLISES DE CAUSALIDADE: APLICAÇÕES DO PROCESS TRACING ${ }^{12}$
}

\author{
Iana Alves de Lima ${ }^{3}$
}

\begin{abstract}
RESUMO
O presente artigo tem como objetivo discutir as possibilidades de utilização da técnica de process tracing na investigação de mecanismos causais em pesquisas sociais. Inicialmente, buscou-se situar a técnica dentro do debate metodológico sobre métodos qualitativos, apresentando as principais críticas direcionadas ao seu uso, especialmente as concernentes à incapacidade de lidar com inferências causais e incipiente rigor metodológico. Em seguida, a partir da revisão de produções teóricas a respeito do tema, objetiva-se apresentar definições do process tracing e apontar para a inadequação das principais críticas à referida técnica. Ademais, serão detalhadas suas principais aplicações e possíveis testes de rigor metodológico. Dessa forma, o artigo apresenta argumentos que sustentam a utilização do process tracing em desenhos de pesquisa que objetivam inferências causais e conclui que a técnica não é uma ferramenta acessória que orbita desenhos de estudos quantitativos, mas um instrumento de pesquisa autônomo passível de testes de rigor metodológico e capaz de fornecer validade.
\end{abstract}

Palavras-chave: process tracing; estudos de pequeno N; inferência causal; métodos qualitativos; rigor metodológico

\begin{abstract}
The present paper aims to discuss the possibilities of using the process tracing technique in the investigation of causal mechanisms in social research. Initially, we sought to situate the technique within the methodological debate on qualitative methods, presenting the main criticisms directed to its use, especially those concerning the inability to deal with causal inferences and incipient methodological rigor. Then, based on the review of theoretical productions about the theme, follows the presentation of definitions of the process tracing and to point out the inadequacy of the main criticisms towards the technique. In addition, it will be detailed its main applications and possible tests of methodological rigor. In this way, the article presents arguments that support the use of process tracing in research designs that target causal inferences and concludes that the technique is not an accessory tool that orbits drawings of quantitative studies, but an autonomous research instrument that can be rigorously tested and capable of providing validity.
\end{abstract}

Keywords: process tracing; small N studies; causal inference; qualitative methods; methodological rigor

\section{RESUMEN}

El artículo tiene como objetivo discutir las posibilidades de utilizar la técnica de process tracing en la investigación de los mecanismos causales en la investigación social. Inicialmente, se trató de colocar la técnica dentro del debate metodológico en métodos cualitativos, con las principales críticas dirigidas a su uso, especialmente en relación con la imposibilidad de hacer frente a inferencias de causalidad y poco rigor metodológico. Luego, a partir de la revisión de los tratados teóricos sobre el tema, se discuten las definiciones del concepto de process tracing y las inadecuación de las principales críticas a esta técnica. Además, serán presentadas sus principales aplicaciones y los posibles testes de rigor metodológico. Por lo tanto, el artículo presenta argumentos que apoyan el uso del process tracing en diseños de investigación que tienen como objetivo inferencias causales y concluye que la técnica no es una herramienta accesoria que orbita investigaciones cuantitativas, sino un instrumento autónomo de investigación que puede ser sujeto a testes metodológicos rigurosos y capaz de proporcionar validad cientifica. Palabras clave: process tracing; estudios de pequeño $\mathrm{N}$; inferencia causal; métodos cualitativos; rigor metodológico

\footnotetext{
${ }^{1}$ Enviado: 16/01/2017

Aceito: $13 / 06 / 2017$

${ }^{2}$ DOI: $10.5380 /$ recp.v8i1.50210

${ }^{3}$ Mestra em Ciência Política pelo Instituto de Ciência Política da Universidade de Brasília. Bacharela em Ciência Política pela mesma instituição.
} 


\section{INTRODUÇÃO}

A escolha de determinada metodologia é decisiva nos resultados de uma pesquisa e precede de questões ontológicas que devem estar claras para o pesquisador. Como destacam Colllier, Brady e Seawright (2004), tanto metodologias quantitativas quanto qualitativas são difíceis de executar. No entanto, as técnicas qualitativas frequentemente são preteridas no debate metodológico. Não é incomum serem apontadas como menos rigorosas e incapazes de gerar explicações válidas para os fenômenos sociais (GERRING, 2004; HEBLING, 2014).

Esse pensamento dominante na Ciência Política acaba por promover uma espiral de desvalorização em que técnicas qualitativas são marginalizadas e ocupam menos espaços de debate para aprimoramento metodológico. Nesse sentido, é necessária a ampliação do debate sobre técnicas qualitativas e o refinamento das mesmas.

Hall (2003) aponta que, muitas vezes, desenhos de pesquisa de $\mathrm{N}$ pequeno são desprezados por terem menor grau de liberdade para estabelecer correlações para inferência causal $^{4}$. Nessa linha, diversas críticas são apresentadas às técnicas utilizadas em estudos de caso. Dentre elas está o process tracing - ou mapeamento de processo -, frequentemente acusado de incapacidade de lidar com inferências causais e de pouco rigor metodológico, o que dificultaria sua diferenciação de narrativas descritivas ou storytelling. Contrapondo-se a essa crítica, parte da literatura sai em defesa da técnica, como o próprio Hall, que afirma que pesquisas de $\mathrm{N}$ pequeno são terreno fértil para process tracing e servem sim para testar proposições causais.

O objetivo deste trabalho é, nessa mesma orientação, argumentar a favor da utilização da técnica de process tracing, buscando contrapor o entendimento de que tal técnica caracterizaria um trabalho "pouco científico" ou de natureza prévia à pesquisa em si. Debateremos aqui como o process tracing diferencia-se de narrativas descritivas, bem como por que é possível garantir rigor metodológico e como é possível sua utilização em estudos que sugerem inferências causais, diferenciando os objetivos de pesquisas de $\mathrm{N}$ pequeno das de $\mathrm{N}$ grande.

A finalidade deste trabalho é mostrar que nem todos os desenhos de pesquisa possuem os mesmos objetivos e que a aplicação de diferentes técnicas fornece dados e informações de distintas naturezas, exigindo, por conseguinte, diferentes julgamentos. O intuito aqui não é

\footnotetext{
${ }^{4}$ Essa é uma das principais críticas de King, Keohane e Verba (1994) à utilização da técnica de process tracing. Para os autores, o pequeno grau de liberdade geraria inferências causais fracas, que permitiriam que o process tracing apenas fosse utilizado em um momento anterior à pesquisa e não como parte do desenho, servindo unicamente ao propósito de aumentar as implicações observáveis do estudo.
} 
negar as limitações expostas por King, Keohane e Verba (1994) -, e outras posteriores, mas sim alterar a ótica da crítica, considerando o alvo diferenciado de cada desenho de pesquisa.

Inicialmente, serão reunidas algumas das principais definições e possibilidades de utilização da técnica de process tracing e em seguida situaremos as críticas e réplicas no debate metodológico sobre causalidade e adequação de estudos qualitativos. Após a discussão sobre a pertinência de estudos de caso na investigação de mecanismos causais, serão apresentadas as principais críticas direcionadas especificamente ao process tracing e, por fim, serão apresentados aprimoramentos metodológicos que possibilitam maior clareza sobre a aplicação prática da técnica e superação de riscos.

\section{DEFININDO PROCESS TRACING E SUAS POSSIBILIDADES}

O termo process tracing, ou mapeamento de processo, surgiu por volta dos anos 1970 em estudos de Psicologia sobre a tomada de decisão dos indivíduos. Ao ser assimilado pela Ciência Política, seu conceito passou a ser mais abrangente, explorando cadeias explicativas de fenômenos sociais e não apenas individuais (BENNETT; CHECKEL, 2015).

A definição de process tracing não é homogênea entre os estudiosos. Collier define a técnica como um exame sistemático de evidências de diagnóstico selecionadas e analisadas à luz de perguntas de pesquisa e hipóteses propostas pelo pesquisador. Seria uma ferramenta analítica para desenhar descrições e inferências causais a partir de "pedaços" de evidências de diagnósticos (COLLIER, 2011a).

Segundo o autor, o process tracing pode ser utilizado para descrever fenômenos políticos e sociais, mas também para avaliar afirmações causais, avaliando ou criando novas hipóteses, examinando mecanismos causais ou mesmo buscando resolver questões de causalidade recíproca.

Bennett e Checkel (2015), por sua vez, definem process tracing como um exame de passos intermediários de um processo para realizar inferências sobre hipóteses de como esse processo gerou determinado resultado. Em ambas as definições fica claro que se trata de uma análise within-cases e que tem como alvo o exame de mecanismos específicos que explicam determinadas relações causais. O objetivo imediato não é a generalização estatística e, portanto, a técnica não deve ser "cobrada" por isso.

$\mathrm{Na}$ tentativa de tornar o conceito de process tracing mais tangível, George e Bennett (2005) apresentaram uma metáfora simples e efetiva para o entendimento da técnica. Quando vemos 50 peças de dominó enfileiradas e caídas sobre uma mesa, podemos dizer que a queda 
da primeira peça causou a queda da última? Para ter certeza de que a quinquagésima peça não caiu por outras causas - movimentos da mesa, alguém deliberadamente a empurrando ou mesmo outro fator que tenha promovido sua queda simultaneamente ou mesmo anteriormente à primeira - precisamos analisar o encadeamento de movimentos das outras peças para verificar se a nossa hipótese está correta - de que a queda da primeira causou a queda da última.

Percebemos que em todas as definições apresentadas está presente a característica de que o process tracing é uma ferramenta analítica e não meramente descritiva. Nesse sentido, Venesson (2008) apresenta três aspectos pelos quais o mapeamento de processos pode se diferenciar de meras narrativas descritivas: é uma técnica focada, é orientada pela teoria e é utilizada na explicação de um caminho causal. Com relação ao primeiro deles, o fato de que é uma técnica focada, entende-se que ele lida seletivamente com alguns aspectos do fenômeno que são relevantes para o estudo em questão, de forma a dialogar com a teoria. $\mathrm{O}$ pesquisador não vai simplesmente a campo considerando todo tipo de estímulo e absorvendo todos os aspectos do contexto que busca examinar.

O segundo atributo é justamente o fato de a técnica ser amparada por um aparato teórico. A utilização do process tracing não é uma congregação de múltiplos fatos aleatórios que o pesquisador apenas por intuição julga relevante para a explicação de fenômenos sociais. Muito pelo contrário, é imperativa sua interação com a teoria desenvolvida sobre o assunto na escolha dos fatores explicativos.

Nesse sentido, Collier (2011a) e Hall (2003) também destacam a importância de um enquadramento teórico para o início da análise de determinado processo. Isso é relevante para que o pesquisador aja minimamente orientado na análise do objeto. No entanto, é importante o cuidado para não perder possibilidades de insights que possam agregar ao estudo pela rigidez de alguma teoria. Uma das grandes vantagens do process tracing em relação a outros métodos é justamente sua possibilidade para novas descobertas e inovação na análise dos fenômenos.

Complementarmente, Bennett e Checkel (2015) apresentam com mais clareza a possibilidade de dedução e indução no mapeamento de processos, garantindo assim a capacidade de inovação para permitir o melhor entendimento dos mecanismos envolvidos no desenrolar dos fenômenos sociais. A abertura do process tracing a novas explicações é uma vantagem por permitir que o pesquisador se relacione de forma diferenciada com seu objeto de estudo, aumentando sua percepção sobre o fenômeno estudado e não implica, de forma alguma, baixo rigor metodológico.

A terceira característica observada por Venesson (2008) é que o objetivo do process tracing é fornecer uma explicação de um caminho causal. Isso mostra a importância dessa 
técnica para além de correlações matemáticas e como ela pode ser utilizada para desvendar mecanismos fundamentais que consolidam teorias explicativas. O process tracing, portanto, diferencia-se de várias maneiras de uma mera narrativa e pode e deve ser utilizado em estudos que objetivam inferências causais, como veremos nas seções seguintes.

\section{A CAUSALIDADE NO DEBATE METODOLÓGICO}

Tem-se observado nos últimos anos um acirramento do debate sobre o uso de métodos quantitativos ou qualitativos na Ciência Política, bem como suas respectivas limitações e

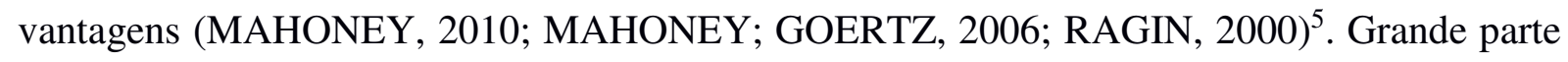
desse debate é marcado pelo posicionamento de pesquisadores adeptos de metodologias qualitativas, em essência estudos de caso, defendendo suas escolhas metodológicas. Essas manifestações buscam ressaltar o potencial explicativo dos estudos de caso, bem como apresentar críticas à tradição norte-americana de estudos quantitativos de $\mathrm{N}$ grande $^{6}$.

Cabe ressaltar que o uso de métodos qualitativos não é uma novidade nas ciências sociais. Pelo contrário, o início das ciências sociais é marcado por narrativas descritivas, etnografias e estudos de caso em política comparada (ERICKSON, 2011). No entanto, tem-se observado uma crescente "revalorização" de estudos qualitativos, com produção de conteúdo metodológico robusto em defesa desses métodos, configurando um momento de "nova metodologia qualitativa" (MAHONEY, 2010).

O leque de discussão é amplo, mas um aspecto central é sobre a capacidade de cada abordagem em realizar inferências causais. Além disso, observa-se uma maior tendência em valorizar estudos que fazem uso de métodos estatísticos e modelos de análise de regressão (HALL, 2003). No entanto, tem sido crescente o avanço de perspectivas pluralistas e multimétodos, o que nos leva a encarar alguns questionamentos.

Uma série de questões que dizem respeito à própria construção do debate de causalidade emergem: as técnicas para análise causal apresentadas pela literatura quantitativista são adequadas a todo problema de pesquisa? Garantir a homogeneidade de unidades e ausência

\footnotetext{
${ }^{5}$ De forma simplificada, trata-se como métodos qualitativos aqueles utilizados em estudos de $\mathrm{N}$ pequeno ou médio. Com relação a métodos quantitativos, entende-se como aqueles que fazem uso de métodos econométricos estatísticos - no tratamento de dados de survey ou outros dados agregados, ou seja, $\mathrm{N}$ grande.

6 As tradições metodológicas norte-americana e europeia foram sensivelmente distintas nas últimas décadas. Enquanto nos Estados Unidos observou-se grande avanço da utilização de métodos quantitativos em detrimento de estudos de $\mathrm{N}$ pequeno, na Europa observava-se a ascensão de métodos mais variados para $\mathrm{N}$ médio e pequeno - com foco, principalmente, na realização de estudos comparados. Recentemente, no entanto, tem-se observado que a tradição europeia aproximou-se da estadunidense (MOSES; RIHOUX; KITTEL, 2005).
} 
de multicolinearidade e endogeneidade entre variáveis (KING; KEOHANE; VERBA, 1994) é o único caminho "seguro" e adequado para um bom desenho de pesquisa e inferências válidas? Métodos estatísticos possibilitam melhores inferências causais? É possível analisar relações de causalidade deslocadas de seu contexto?

A tradição quantitativista, que tem como expoente central a obra de King, Keohane e Verba (1994), vai na direção de uma certa supremacia dos métodos estatísticos. No entanto, outra corrente de pesquisadores afirma que, muitas vezes, estudos de $\mathrm{N}$ grande que se utilizam de técnicas estatísticas violam os próprios princípios que estabelecem para validade científica (MAHONEY, 2001), além de ter limitada capacidade de descoberta científica por suas estruturas rígidas de desenho (RAGIN, 2000).

Dentre os adeptos da tradição quantitativista, grande parte das críticas em direção aos desenhos de pesquisa de pequeno $\mathrm{N}$ giram em torno de questões de validade, viés de seleção e capacidade de generalização das análises geradas a partir de estudos de caso (REZENDE, 2011). Com relação à validade, Rezende (2011) destaca que estudos de caso são a escolha mais acertada para desvendar mecanismos causais de fenômenos sociais, justamente pela densidade interpretativa que o método oferece.

Nessa mesma direção, Hall (2003) aponta que, cada vez mais, os pesquisadores enxergam que nem tudo pode ser explicado por regressões. Dentre as principais críticas estão a incapacidade de explicar por que os fatores explicativos relacionam-se com o fenômeno estudado, a discordância com a premissa de que sem regularidade empírica não há causalidade, além da inabilidade em explicar o black box, conceito proposto para resumir as conexões muitas vezes não explorados entre X e Y (ELSTER, 1998; MAHONEY, 2001).

Há ainda os apontamentos sobre como métodos correlacionais acabam ignorando a distinção entre causas necessárias e suficientes, além de não problematizarem as populações escolhidas, o que poderia incluir casos heterogêneos no estudo (MAHONEY, 2001). A inclusão de casos heterogêneos violaria a própria premissa de homogeneidade das unidades invocada pelos quantitativistas.

Nesse sentido, uma correlação apresentada em modelos estatísticos seria a identificação de um padrão de covariância entre uma causa e um efeito, enquanto a exploração dos mecanismos causais ocupa-se de conectar processos explicativos entre as supostas causas e efeitos (HÉRITIER, 2008). Ou seja, os mecanismos causais explicariam por que ocorre uma correlação em determinado modelo, questão central para a análise de fenômenos sociais e que, essencialmente, demanda análises qualitativas - de pequeno $\mathrm{N}$ - de investigação. 
Mas o que seriam exatamente os mecanismos causais de um fenômeno? McAdam, Tarrow e Tilly (2001) trazem uma definição de mecanismos causais a partir de uma cadeia explicativa de determinado acontecimento. Para os autores, mecanismos causais são eventos que alteram a relação entre determinados elementos em condições similares e em situações distintas. A sequência desses mecanismos formam processos que explicam episódios.

Falleti e Lynch (2009) apresentam definição similar de que mecanismos causais são conceitos que explicam como e por que uma causa hipotética contribui para um resultado em determinado contexto. Essa definição é interessante pela percepção de que explicações causais válidas dependem não só da análise desses mecanismos como também do contexto em que o fenômeno está inserido, independentemente do método utilizado.

A especificação do contexto é relevante, pois confronta o pressuposto de homogeneidade de unidade, anteriormente mencionado. Tal premissa seria problemática, uma vez que unidades homogêneas podem comportar-se de maneira distinta em diferentes contextos. Nesse sentido, estudos de caso alavancam as explicações sobre determinados fenômenos, já que se ocupam da influência dos elementos do contexto de cada caso.

Definir com clareza o que é um mecanismo causal é fundamental, pois permite ao pesquisador compreender o objeto em estudo, bem como propor um desenho de pesquisa alinhado com os objetivos propostos. Com relação a isso, retomando um dos argumentos de Elster sobre as precauções ao delimitar mecanismos causais, essa maior clareza contribuiria para diferenciar explicações causais de storytelling, por exemplo, situação em que não haveria rigor metodológico (ELSTER, 1998).

Considerando os pontos apresentados e retomando o debate inicial, não é possível dizer que uma abordagem quantitativa é superior a uma qualitativa em termos de cientificidade. Cada uma tem suas limitações e sua aplicação está relacionada ao problema que se pretende estudar, não implicando uma escolha excludente de metodologias - muito pelo contrário.

No entanto, cabe discutir mais as possibilidades de pontos de convergência em um desenho de pesquisa, bem como as reais condições de interação entre os pesquisadores de cada abordagem, uma vez que tanto os métodos qualitativos quanto quantitativos estão cada vez mais sofisticados, implicando muitas vezes na especialização dos acadêmicos, o que poderia conduzir naturalmente a uma limitação do escopo de pesquisa (DELLA PORTA; KEATING, 2008). 


\section{O DEBATE EM TORNO DA UTILIZAÇÃO DO PROCESS TRACING: CRÍTICAS E CONTRAPONTOS}

Os estudos que elegem o process tracing para seus desenhos de pesquisa enfrentam diversas críticas na Ciência Política. Parte dessas críticas é abrangente, direcionada a estudos de caso em geral e outra parte é direcionada especificamente à técnica. Dentre as mais genéricas, é possível dizer que o mapeamento de processo não supera duas grandes críticas levantadas pelos adeptos da tradição quantitativista: a capacidade de realizar estimativas, construindo inferências causais com capacidade de generalização (KING; KEOHANE; VERBA, 1994) e o viés de seleção presente em estudos de pequeno N (COLLIER; MAHONEY, 2011).

E de fato não superam. Contudo, cabe destacar que, muitas vezes, essas são críticas equivocadas aplicadas indiscriminadamente a todo desenho de pesquisa pela ótica quantitativista. Equivocadas não no sentido de que esses problemas não ocorrem ao utilizarmos a técnica de process tracing, mas que essas críticas confundem a natureza do dado que se busca coletar e supervalorizam requisitos relevantes a outro desenho de pesquisa que não o qualitativo. Os problemas levantados nessas críticas não invalidam o estudo e muitas vezes fazem parte de trade-offs que o pesquisador precisa fazer sobre qual problema ele combaterá em um desenho qualitativo (COLLIER; MAHONEY, 2011).

O objetivo de um estudo que lança mão de process tracing é a compreensão em profundidade de determinados mecanismos causais e de como o processo de causalidade é observado na realidade e não necessariamente a generalização de resultados a partir de múltiplas observações. Esse objetivo de pesquisa, em geral, não é o mesmo do de trabalhos que realizam inferências estatísticas. Isso não invalida nenhum dos dois métodos ou diminui sua importância no aparato metodológico disponível aos cientistas sociais.

Ademais, a crítica de que um estudo de caso não pode promover generalizações não é completamente verdadeira. A partir do teste de "falsificação", por exemplo, é possível identificar casos desviantes que comprometem a generalização de alguma teoria, o que em si já é um resultado generalizável. Um exemplo clássico é o teste de Karl Popper sobre a afirmativa “Todos os cisnes são brancos". Nesse caso, a identificação de um único cisne negro já comprometeria a hipótese, gerando um conhecimento novo de amplitude geral (FLYVBJERG, 2011). Para essas conclusões não foram necessárias múltiplas observações de unidades homogêneas, mas apenas a análise de um único caso desviante selecionado de maneira focada e não aleatória. 
Em uma tentativa conciliatória, Vennesson (2008) afirma que o process tracing é capaz de atender tanto expectativas positivistas quanto interpretativistas. Com relação às primeiras, a técnica examina tanto se o processo causal da teoria pode ser observado empiricamente quanto se a escolha de variáveis independentes e explicativas é adequada e se os dados são confiáveis. Para os últimos, sua principal vantagem seria a possibilidade de análise do contexto no qual os fenômenos ocorreram e da relação entre crenças e comportamentos dos atores.

Essa discussão remete a debates ontológicos e em que medida os pesquisadores concordam no que constitui a pesquisa científica social. Dessa forma, os diferentes métodos utilizados também refletem os objetivos de cada pesquisa, que variam dependendo da abordagem do pesquisador.

Nesse aspecto, Collier, Brady e Seawright (2004) fazem uma importante contribuição diferenciando dois tipos distintos de observação. A primeira, data-set observation (DSO), é um conjunto de dados que utiliza a mensuração estatística de variáveis para explicação de casos e seria proveniente de abordagens mais quantitativistas. A outra, casual-process observation $(C P O)$, seria um "pedaço" dos dados que fornecem informações aprofundadas de contexto ou mecanismos que contribuem para a alavancagem da inferência causal, sendo as observações obtidas por meio de process tracing (MAHONEY, 2010).

Essa diferenciação é relevante porque contribui para o entendimento de que o process tracing de fato não deve ser utilizado com o propósito de generalização idealizado por King, Keohane e Verba

Nesse sentido, o process tracing surge como uma preciosa ferramenta na investigação de inferências causais, não no aspecto da generalização estatística, mas na exploração de mecanismos causais. Esse entendimento vai ao encontro do argumento defendido neste trabalho de que a análise em profundidade desses mecanismos e de seus desdobramentos na realidade social são de importância seminal para a consolidação de teorias explicativas.

Com relação às críticas específicas ao process tracing, pode-se dizer que, em geral, elas dizem respeito à pouca "cientificidade" do método. Como Bennett e Checkel (2015) apontam, analisar processos históricos na tentativa de identificar mecanismos causais é muito mais intuitivo do que a utilização de modelos estatísticos na explicação de fenômenos sociais.

Com isso, para além do risco de a ferramenta reduzir-se ao storytelling - perdendo, assim, capacidade analítica -, há ainda o risco de a baixa sistematização do mapeamento levar a erros de inferência que comprometem as conclusões do estudo. Nesse sentido, para prevenir 
essas falhas é necessário garantir rigor metodológico por meio de testes e aplicação sistematizada da ferramenta. Esses pontos serão explorados na seção abaixo.

\section{APLICAÇÕES E TESTES}

Como discutido anteriormente, atualmente experimentamos um momento de "nova metodologia qualitativa", com sensível aumento das produções sobre o uso de estudos de pequeno N (MAHONEY, 2010). Entretanto, quando nos debruçamos sobre a literatura sobre process tracing, muitas vezes não fica claro como podemos utilizar na prática essa ferramenta e como superar os riscos inerentes à sua utilização. Nesse sentido, serão apresentados a seguir alguns trabalhos que vem explorando cada vez mais esse campo e nos auxiliam a clarear as ideias sobre as possibilidades da técnica.

Beach e Pedersen (2013) lapidam a definição de mapeamento de processo, desagregando a técnica em três variações práticas, considerando as diferentes maneiras em como a técnica interage com a teoria e suas diferentes aplicações. De acordo com autores, o process tracing pode ser voltado para o teste de teorias, para a construção de teorias ou para a explicação de resultados específicos. Os autores detalham como analisar mecanismos causais em cada tipo de variação, fornecendo uma fonte mais clara de onde e quando utilizá-lo ${ }^{7}$.

Com relação ao tipo de dado obtido com process tracing e sua utilidade para testes de teoria, Mahoney (2010) vai mais a fundo no esclarecimento sobre casual-process observation - CPO. Para o autor existem três tipos distintos de CPOs utilizados para testar teorias: CPO de variável independente, $\mathrm{CPO}$ de mecanismo e CPO de resultados auxiliares.

O primeiro tipo é útil em fenômenos nos quais a própria existência da variável independente é questionada, ou seja, as possíveis causas são controversas. Nesse sentido, um CPO de variável independente seria um conjunto de dados que reforça a plausibilidade da escolha da variável explicativa. O CPO de mecanismo, por sua vez, gera informação sobre eventos intermediários nas cadeias causais. Segundo Mahoney (2010), a alavancagem promovida por esse tipo de observação não diz respeito ao maior número de eventos observáveis, mas à capacidade de as observações individuais reforçarem ou desafiarem as premissas do pesquisador.

Um exemplo clássico trazido pelo autor é o trabalho States and Social Revolutions, de Theda Skocpol, que conclui que movimentos progressistas de cunho ideológico não são causas

\footnotetext{
${ }^{7}$ Ver quadro-resumo 2.1 (BEACH; PEDERSEN, 2013, p. 21).
} 
importantes de revoluções sociais. Isso porque, a partir da utilização do process tracing, a pesquisa não conseguiu identificar o principal mecanismo intermediário que sustentaria a existência dessa causa, que seria a atuação desses movimentos em mobilizar e apoiar insurreições das classes urbanas e rurais mais baixas. Na verdade, mostrou-se que, em geral, esses movimentos nascem posteriormente ou contemporaneamente às próprias revoltas, não sendo, portanto, o fator causativo delas (MAHONEY, 2010).

Os CPO's de resultados auxiliares, diferentemente dos anteriores, não apontam a existência de causas ou os mecanismos intermediários que conectam a cadeia causal. Eles são resultados acessórios esperados pelas causas que geraram o fenômeno em análise, ou seja, seriam uma espécie de "rastro" deixado pelas variáveis explicativas e que reforçam a plausibilidade da teoria.

Com relação à capacidade explicativa, Collier (2011a) aponta que o process tracing é uma valiosa ferramenta tanto em inferências descritivas quanto em inferências causais ${ }^{8}$. Com relação à primeira, base fundamental a identificação de causalidade, o autor apresenta alguns exemplos de estudos que foram bem-sucedidos no uso da técnica.

Um valioso exemplo é o estudo de Tannenwald (1999) sobre o "Tabu Nuclear". Na tentativa de explicar os processos que levaram às políticas de controle nuclear nos Estados Unidos, Tannenwald identifica uma variável independente crucial da explicação dessas diretrizes normativas, o tabu nuclear gerado após a Segunda Guerra Mundial. O rigor do estudo sustenta-se nas provas empíricas apresentadas de que uma reação pública contrária ao uso de armas nucleares, de fato, existiu; o detalhamento de qual foi sua dimensão; e que os elementos dessa reação massiva contribuíram para o surgimento de um tabu (COLLIER, 2011a).

Outro grande exemplo de inferência descritiva, esse no campo dos estudos legislativos, é o trabalho de Fenno (1977) sobre o relacionamento de congressistas estadunidenses e suas constituencies. A partir de entrevistas e observação participante em viagens de congressistas aos seus distritos de origem - técnicas as quais o autor resume em "soaking and poaking" -, Fenno propõe uma tipologia de estilos de representação e tipos de constituencies que guiou uma série de trabalhos posteriores (COLLIER, 2011a).

Uma boa descrição é parte fundamental das análises de fenômenos sociais, mas é no desafio de realizar inferências causais que o uso do process tracing enfrenta seus maiores

\footnotetext{
${ }^{8}$ Ambas as definições são compreendidas conforme o proposto por KKV (1994), sendo a inferência descritiva uma análise que supera a mera apresentação dos dados coletados, propondo uma investigação sistematizada e organização lógica das evidências levantadas (COLLIER, 2011a).
} 
percalços. Nesse sentido, um importante aprimoramento na utilização do mapeamento de processos é com relação aos testes empíricos de inferências causais.

Collier (2011b), por exemplo, elenca quatro testes que poderiam ser utilizados para avaliar a inferência causal: Straw-in-the-Wind, Hoop, Smoking-Gun e Doubly-Decisive, em ordem gradativa de sua capacidade de confirmar hipóteses. Os testes são distribuídos em um quadrante que indica a capacidade de identificar causas suficientes e necessárias para a inferência sobre determinado evento, ou seja, a depender do teste que passar, uma causa pode ser classificada como suficiente ou necessária para a ocorrência de determinado fenômeno.

Por exemplo, o teste Straw-in-the-Wind não é decisivo na confirmação de uma hipótese, mas é um indicativo que fortalece sua plausibilidade, enquanto o Doubly-Decisive não só confirma a hipótese como elimina as demais. Esses testes demandam grande capacidade analítica do pesquisador e envolvem encadeamento lógico de ideias e informações, o que requer uma investigação aprofundada do processo em questão.

Para a ilustração do uso dos testes, Collier (2011a) utiliza o conto literário "Silver Blaze", sobre uma investigação de Sherlock Holmes a respeito do desaparecimento de um cavalo de corrida e a morte de seu treinador por um forte golpe na cabeça. Após a análise descritiva do cenário, Holmes levanta três hipóteses: (1) o treinador suicidou-se; (2) um dos assistentes o assassinou; ou (3) o cavalo provocou os ferimentos que causaram a morte do treinador.

$\mathrm{Na}$ averiguação das provas, uma evidência considerada como Straw-in-the-Wind seria o fato de que o cão de guarda do estábulo não fez nenhum alarde na noite do crime, o que não é causa necessária nem suficiente para o apontamento do autor do crime, mas confirma a hipótese de que ele é alguém que circulava pelo estábulo e conhecia o cão, reduzindo a lista de suspeitos e adicionando plausibilidade às hipóteses propostas. Essa observação poderia, inclusive, ser identificada como um CPO de resultados auxiliares, já que o comportamento do cachorro não aponta a causa em si, mas revela um "rastro" deixado por ela.

Um teste Hoop, por sua vez, apresentaria um critério necessário, porém não suficiente para a explicação causal. No caso em análise, dentre os suspeitos identificados, uma evidência era de que o assistente tinha uma arma em potencial, o que faria a hipótese de que ele era autor do crime passar no teste Hoop por ser um elemento necessário.

Essa hipótese, no entanto, falha em um segundo teste Hoop que confronta a evidência de que esse sujeito teria um perfil pacífico e não teria a força física suficiente para deferir o golpe que causou a morte do treinador. O teste Hoop também descartaria a hipótese de suicídio, dada a improbabilidade de o treinador ter causado um ferimento tão grave em si mesmo. 
Um teste Smoking-Gun, como o nome sugere, implicaria incriminar um sujeito flagrado com a arma do crime em ação ou logo após o ocorrido. Isso significa que uma pessoa flagrada nessa situação poderia ser considerada culpada. Porém, na ausência dessa evidência, não se pode considerar alguém inocente simplesmente por não ser pego em flagrante. Ou seja, esse teste identifica critérios suficientes, mas não necessários na inferência causal, já que o fato de não passar no teste - não ser pego com a arma em flagrante - não rejeita a hipótese incriminação de determinado suspeito. Nesse caso, seriam necessárias evidências adicionais para reforçar a hipótese.

O teste Doubly-Decisive, que apresentaria critérios necessários e suficientes, é raro nas ciências sociais (COLLIER, 2011a), mas pode ser verificado pela combinação de outros testes na eliminação de hipóteses rivais. No caso em análise, como a primeira e a segunda hipóteses foram eliminadas por testes Hoop, e o teste Straw-in-the-Wind não indicou a existência de uma pessoa nova no cenário do crime, Holmes concluiu que o próprio cavalo causou os ferimentos que levaram à morte do treinador.

Esses testes demandam do pesquisador hipóteses claras e bem especificadas, conhecimento de contexto e capacidade de encadeamento lógico de ideias. Tanto a aplicação dos testes quanto o entendimento das variações do process tracing dependem em grande medida da capacidade interpretativa do pesquisador e também de certa expertise. Isso não quer dizer que a técnica seja sustentada por intuição do pesquisador, mas conta com a sua bagagem da mesma maneira que estudos estatísticos também o exigem na operacionalização da teoria, escolha de variáveis, entre outros.

\section{CONCLUSÃO}

O propósito desse trabalho foi apresentar argumentos que sustentam a utilização de process tracing em desenhos de pesquisa que objetivam inferências descritivas ou inferências causais. Buscou-se, inicialmente, situar a técnica no debate metodológico sobre métodos qualitativos, apresentando as principais críticas e contraposições a elas e a adequação da perspectiva de seus principais julgamentos.

A partir de produções recentes que configuram um momento de "nova metodologia qualitativa" (MAHONEY, 2010), foram organizados os principais argumentos da literatura a respeito da diferença entre o tipo de dado obtido em estudos de pequeno $\mathrm{N}$ daqueles de grande $\mathrm{N}$ e das vantagens de estudos de caso na investigação de mecanismos causais. 
Corroborou-se ao entendimento de que o process tracing fornece dados distintos dos resultantes de estudos estatísticos e que os mesmos são de fundamental importância para a alavancagem de inferências causais e consolidação de teorias.

O process tracing, portanto, não é uma ferramenta acessória que orbita desenhos de estudos quantitativos. Ele caracteriza-se como uma técnica que contém suas especificidades e limitações, fornece validade, é passível de testes de rigor metodológico e tem sido cada vez mais refinado pela comunidade acadêmica, garantindo um lugar protagonista no aparato metodológico disponível aos cientistas sociais.

\section{REFERÊNCIAS}

BEACH, D.; PEDERSEN, R. B. Process-Tracing Methods. Foundations and Guidelines. Ann Arbour: University of Michigan Press, 2013.

BENNETT, A.; CHECKEL, J. T. Process tracing: from philosophical roots to best practices. In: BENNETT, A.; CHECKEL, J. T. (Eds.). Process tracing. From Metaphor to Analytic Tool. Cambridge: Cambridge University Press, 2015.

COLLIER, D. Understanding Process Tracing. PS: Political Science \& Politics, v. 44, n. 4, p. 823-830, 2011a.

COLLIER, D. Teaching Process Tracing: Examples and Exercises. PS: Political Science \& Politics, v. 44, n. 4, p. 823-830, 2011 b.

COLLIER, D.; BRADY, H. E.; SEAWRIGHT, J. Sources of Leverage in Causal Inference: Toward an Alternative View of Methodology. In: BRADY, H. E.; COLLIER, D. (Eds.). Rethinking Social Inquiry: Diverse Tools, Shared Standards. Lanham, MD: Rowman and Littlefield Publishers, 2004. p. 229-266.

COLLIER, D.; MAHONEY, J. Insights and Pitfalls: Selection Bias in Qualitative Research. World Politics, v. 49, n. 1, p. 56-91, 2011.

DELLA PORTA, D.; KEATING, M. Approaches and Methodologies in the Social Sciences A pluralist perspective. Cambridge: Cambridge University Press, 2008.

ELSTER, J. A Plea for Mechanisms. In: HEDSTROM, P.; SWEDBERG, R. (Eds.). Social Mechanisms: An Analytical Approach to Social Theory. New York: Cambridge University Press, 1998. v. 57p. 45-71.

ERICKSON, F. A History of Qualitative Inquiry in Social and Educational Research. In: DENZIN, N. K.; LINCOLN, Y. S. (Eds.). The SAGE Handbook of Qualitative Research. 4th. ed. [s.1.] SAGE Publications, 2011. p. 43-59.

FAllet, T. G.; LYNCH, J. F. Context and Causal Mechanisms in Political Analysis Comparative Political Studies, 2009. 
FENNO, R. F. U.S. House Members in Their Constituencies: An Exploration. The American Political Science Review, v. 71, n. 3, p. 883-917, 1977.

FLYVBJERG, B. Case study. In: DENZIN, N. K.; LINCOLN, Y. S. (Eds.). The Sage Handbook of Qualitative Research. 4th. ed. CA: Sage: Thousand Oaks, 2011. p. 301-316.

GEORGE, A.; BENNETT, A. Case Studies and Theory Development in the Social Sciences. London/Cambridge: MIT Press, 2005.

GERRING, J. What Is a Case Study and What Is It Good for? American Political Science Review, v. 98, n. 2, p. 341-354, 2004.

HALL, P. A. Aligning ontology and methodology in comparative research. In: MAHONEY, J.; RUESCHEMEYER, D. (Eds.). Comparative historical analysis in the social sciences. Cambridge: Cambridge University Press, 2003. p. 373-404.

HEBling, M. L. Estudo De Caso Em Ciência Política. Agenda Política, v. 2, n. 2, p. 70-83, 2014.

HÉRITIER, A. Causal explanation. In: DELlA PORTA, D.; KEATING, M. (Eds.). Approaches and Methodologies in the Social Sciences. Cambridge: Cambridge University Press, 2008. p. 61-79.

KING, G.; KEOHANE, R. O.; VERBA, S. Designing Social Inquiry. Princeton: Princeton University Press, 1994.

MAHONEY, J. Review essay: Beyond correlational analysis: Recent innovations in theory and method. Sociological Forum, v. 16, n. 3, p. 575-593, 2001.

MAHONEY, J. After KKV: The New Methodology of Qualitative Research. World Politics, v. 62, n. 1, p. 120-147, 2010.

MAHONEY, J.; GOERTZ, G. A Tale of Two Cultures: Contrasting Quantitative and Qualitative Research. Political Analysis, n. 14, p. 227-249, 2006.

MCADAM, D.; TARROW, S.; TILLY, C. Dynamics of Contention. Cambridge: Cambridge University Press, 2001.

MOSES, J.; RIHOUX, B.; KITTEL, B. Mapping political methodology: reflections on a european perspective. European Political Science, v. 4, p. 55-68, 2005.

RAGIN, C. Fuzzy-Set Social Science. Chicago: University of Chicago Press, 2000.

REZENDE, F. DA C. Razões emergentes para a validade dos estudos de caso na ciência política comparada. Revista Brasileira de Ciência Política, v. 6, p. 297-337, 2011.

TANNENWALD, N. The Nuclear Taboo: The United States and the Normative Basis of Nuclear Non-Use. International Organization, v. 53, n. 3, p. 433-468, 1999. 
LIMA, I. A. Técnicas qualitativas em análises de causalidade: Aplicações do Process Tracing

VENESSON, P. Case studies and process tracing: theories and practices. In: DELLA PORTA, D.; KEATING, M. (Eds.). Approaches and Methodologies in the Social Sciences. Cambridge: Cambridge University Press, 2008. 